\title{
Sensibilidad antimicrobiana y caracterización de cepas de Streptococcus pyogenes aisladas de un brote de escarlatina
}

\author{
A lberto González Pedraza-Avilés, Biól, ${ }^{(1,2)}$ C atalina 0 rtiz-Zaragoza, Q BP, ${ }^{(2)}$ \\ Ricardo Mota-Vázquez, Biól, ${ }^{(1)}$ Ma Eloísa Dickinson-Bannack, D ra, ${ }^{(1,2)}$ Rocío D ávila-M endoza, Q BP, ${ }^{(2)}$ \\ Miguel Angel Fernández-O rtega, $\mathrm{Dr}^{(1,2)}$
}

González Pedraza-AvilésA, Ortiz-Zaragoza C, Mota-Vázquez R, Dickinson-Bannack ME,

Dávila-Mendoza R, Fernández-Ortega MA. Sensibilidad antimicrobiana y caracterización de cepas de Streptococcus pyogenes aisladas de un brote de escarlatina. Salud Publica Mex 2002;44:437-441. El texto completo en inglés de este artículo está disponible en: http://www.insp. mx/salud/index.html

\section{Resumen}

Objetivo. Evaluar la actividad in vitro de 13 antibióticos contra 47 Streptococcus pyogenes grupo A (SGA). Determinar la presencia de genes que codifican para exotoxina pirogénica estreptocóccica A (SpeA) y serotipos con base en proteína M. Material y métodos. Estudio transversal hecho en el Centro de Salud Dr. José Castro Villagrana sobre un brote de escarlatina en el Colegio Espíritu deAmérica, entre diciembre de 1999 y enero de 2000. El número de niños estudiados fue 137. Se extrajeron porcentajes de sensibilidad. La concentración inhibitoria mínima (C IM) se obtuvo por microdilución semiautomatizada. Se utilizó un secuenciador automatizado de DN A para el análisis de variación de secuencias en los genes que codifican para proteína M y SpeA. Resultados Todas las cepas fueron sensibles a beta-lactámicos y clindamicina; $12.7 \%$ fueron resistentes a eritromicina. El serotipo M2 fue el más frecuente, 27 del total. Prácticamente todas las bacterias $(96 \%)$ con el gen SpeA tienen el gen que codifica para el serotipo M2. Conclusiones Debido a la reciente reaparición de infecciones
González Pedraza-AvilésA, Ortiz-Zaragoza C, Mota-Vázquez R, Dickinson-Bannack ME,

Dávila-Mendoza R, Fernández-Ortega MA.

Antimicrobial sensitivity and typing

of Streptococcus pyogenes strains isolated during

a scarlet fever outbreak.

Salud Publica Mex 2002;44:437-441.

The English version of this paper

is available at: http://www.insp.mx/salud/index.html

\section{A bstract}

Objective. To evaluate the in vitro activities of 13 antimicrobial agents against 47 group A Streptococcus pyogenes (GAS) strains, and to determine the presence of genes encoding streptococcal pyrogenic exotoxin A (SpeA) and the $M$ - protein serotypes. Materials and Methods. A crosssectional study was conducted at Centro de Salud Dr. José Castro Villagrana, during a scarlet fever outbreak occurring between December 1999 and January 2000, among 137 children at Colegio Espíritu de América. Minimum Inhibitory Concentrations (MICS) were obtained by the semiautomated microdilution method.Automated DN A sequencing was used for analysis of sequence variation in genes enco $d-$ ing the M protein, and SpeA. Results. All strains were sensitive to betalactams and clindamycin. Six (12.7\%) were resistant to erythromycin. The M2 type was the most frequently isolated GAS (27); almost all (96\%) bacteria with the SpeA gene had the gene encoding the M2 protein. Conclusions The recent resurgence of GAS infections calls for molecular epidemiology research and studies on the sensi-

El estudio fue presentado como trabajo en Cartel dentro de las actividades académicas del X Congreso de la Asociación Panamericana de Infectología, XXVI Congreso de laA sociación Mexicana de Infectología y Microbiología C línica,VI Congreso de laA sociación Mexicana para el Estudio de las Infecciones N osocomiales y el II Congreso Panamericano de SIDA, que se llevaron a cabo del 1 al 5 de mayo de 2001 en Guadalajara, Jalisco, México.

(1) Centro de Salud. Doctor José Castro-Villagrana. Servicios de Salud Pública del Distrito Federal. México, D.F., México.

(2) Departamento de Medicina Familiar. Facultad de Medicina. Universidad N acional Autónoma de México. México, D.F., México.

Fecha de recibido: 6 de junio de 2001 • Fecha de aprobado: 28 de mayo de 2002

Solicitud de sobretiros: Biól. Alberto González Pedraza-Avilés. Centro de Salud D octor José Castro Villagrana, SSA/UN AM.

Carrasco y Coapa s/n, colonia Toriello Guerra, 14050,Tlalpan, México, D.F., México.

Correo electrónico: silviala@ servidor.unam.mx 
por SGA se sugiere realizar estudios tanto de sensibilidad a macrólidos y beta-lactámicos, como de epidemiología molecular. El texto completo en inglés de este artículo está disponible en: http://www.insp.mx/salud/index.html

Palabras clave: escar latina; Strepto coccus pyo genes; proteína $M$; exotoxina pirogénica estreptocóccica A; susceptibilidad antimicrobiana; M exico tivity to macrolides and beta-lactams.The English version of this paper is available at:http://www.insp.mx/salud/index.html

Key words: scarlet fever; Strepto coccus pyogenes; M-serotype; streptococcal pyrogenic exotoxin A; susceptibility; Mexico
$S$ e reconoce al Streptococcus beta hemolítico grupo $S$ A (SGA) (Streptococcus pyogenes) como el principal agente etiológico de la faringoamigdalitis aguda. También se asocia con una variedad de procesos infecciosos de localización diversa, entre los que destacan, por su frecuencia, otitis, celulitis, sinusitis, infecciones cutáneas, bacteremia, etcétera; y por su gravedad, fascitis necrozante y choque tóxico, además de producir indirectamente fiebre reumática y glomerulonefritis. En las últimas dos décadas se ha reportado un incremento de las infecciones tanto leves como graves debidas a esta bacteria. ${ }^{1,2}$ Según diversos estudios, lo anterior está en relación con la emergencia de serotipos como M1, M3 y M18 con un mayor potencial invasivo y un aumento en los factores de virulencia, en particular de los proteasas y exotoxinas pirogénicas (Spe,s), 3,4 así como de la exotoxina pirogénica estreptocóccica tipo A, una proteína de $25800 \mathrm{KD}$ que actúa como superantígeno y que se asocia con síndrome de choque tóxico, celulitis, fascitis necrozante y escarlatina. ${ }^{5}$ Además, se observa un menor número de aislamientos de serotipos M4 y M12 que no se asocian con enfermedades invasivas.

Aunque SGA sigue siendo marcadamente sensible a la penicilina, se ha detectado un número cada vez más importante de fallas en el tratamiento con este antibiótico. ${ }^{6,7}$ Existe un aumento en la resistencia frente a los macrólidos y a la clindamicina, utilizados en casos de fallas, alergia e infecciones invasivas, con valores de resistencia a eritromicina que varían desde $12 \%$ en cepas aisladas en Berlín, ${ }^{8}$ hasta $78 \%$ en las de Taiwan. ${ }^{9}$ A su vez, la literatura reporta variantes en la actividad in vitro del SGA contra otros antibióticos beta-lactámicos utilizados también como alternativas de tratamiento. ${ }^{10}$

Con base en un brote de escarlatina presentado en un internado del sur de la Ciudad de México, los objetivos del estudio fueron determinar la presencia de SGA, la sensibilidad antimicrobiana por el método Bauer-Kirby de los SGA aislados, la concentración inhibitoria mínima (CIM) por microdilución automatizada, su serotipo con base en proteína $\mathrm{M}$ y, finalmente, la presencia de la exotoxina pirogénica estreptocóccica A (SpeA) asociada con la aparición de escarlatina.

\section{Material y métodos}

Se presentaron cuatro casos de escarlatina en un internado del sur de la Ciudad de México, dándose tratamiento curativo a éstos y tratamiento preventivo a 16 contactos directos. El tratamiento consistió, en los casos de escarlatina, en penicilina procaínica 800000 U/día por 10 días, más una sola dosis de bencetazil $\mathrm{y}$, para los contactos, bencetazil, una dosis única de $600000 \mathrm{U}$. A pesar de esto, se desarrollaron ocho nuevos casos, cuatro de ellos fueron niños con tratamiento preventivo, por lo que se procedió a tomar cultivo de exudado faríngeo a 137 niños de entre tres y 17 años, 12 de ellos con cuadro clínico de escarlatina; se aislaron 47 SGA, nueve de casos de escarlatina y 38 de contactos.

Identificación. Esta se hizo con base en la morfología colonial y beta hemólisis en gelosa sangre de borrego a $5 \%$, prueba de catalasa y sensibilidad a la bacitracina a $0.04 \%$, y se confirmó por la presencia del antígeno de grupo A de Lancefield con la técnica comercial de aglutinación en látex Slidex Strepto-Kit (Biomériux, Francia).

Pruebas de sensibilidad. Para la determinación de la concentración inhibitoria mínima (CIM) se utilizó el método de microdilución en caldo, Microscan dried gram positive (Dade Behring) siguiendo las instrucciones del fabricante. Al mismo tiempo, se determinó la sensibilidad de las mismas cepas por difusión en agar con disco de Bauer-Kirby, según recomendaciones del National Commitee for Clinical Laboratory Standars (NCCLS). ${ }^{11,12}$ El medio utilizado fue agar de Mueller-Hinton (BBL, México) suplementado con $5 \%$ de sangre de borrego (Microlab). Las placas se incubaron a $35^{\circ}-37^{\circ} \mathrm{C}$ por 24 horas en atmósfera de $5 \%$ de $\mathrm{CO}_{2}$.

Se utilizaron como cepas control las tipo Staphylococcus aureus ATCC 29 213, Escherichia coli ATCC 
25922 y Streptococcus pyogenes ATCC 10 389. La CIM se definió como la más baja concentración que inhibe el crecimiento visible del microrganismo.

Amplificación y secuenciación del gen emm. Esta se realizó en el Departamento de Salud Pública de la Facultad de Medicina de la Universidad Nacional Autónoma de México, en el laboratorio de Patología Molecular. El DNA cromosomal de cada cepa fue obtenido mediante la técnica de extracción con fenol-cloroformo. ${ }^{13}$ El gen emm (proteína M) fue amplificado utilizando los iniciadores descritos por Whatmore. ${ }^{14,15}$ Reacción en cadena de la polimerasa. Se realizó en un termociclador Perkin-Elmer, Modelo 9 700, bajo las siguientes condiciones: desnaturalización $94{ }^{\circ} \mathrm{C} / 90 \mathrm{~s}$, alineamiento $55^{\circ} \mathrm{C} / 90$ s y extensión $72^{\circ} \mathrm{C} / 120$ s durante 30 ciclos. Las estrategias para el análisis de la variación de la secuencia del gen emm han sido previamente descritas. ${ }^{14,15}$ A partir del amplificado del gen emm, la secuencia de nucleótidos del extremo $5^{\prime}$ fue determinada mediante la técnica de dideoxiterminal (Sanger). Las secuencias fueron obtenidas en un secuenciador automatizado de DNA Applied Biosystems, Modelo 310. Un fragmento de la región hipervariable de aproximadamente 150 nucleótidos fue analizado electrónicamente con los programas EDITSEQ, y MEGALING (DNASTAR, Madison, Wis), y comparados con las secuencias descritas para la proteína M. ${ }^{14,15}$

\section{Resultados}

Los resultados de sensibilidad antimicrobiana se presentan en los cuadros I y II; no hubo diferencias significativas entre las dos técnicas utilizadas. No se presentaron resistencias a beta-lactámicos; se presentaron seis cepas $(12.7 \%)$ resistentes a eritromicina y el mismo número con sensibilidad intermedia a clindamicina, así como cuatro $(8.5 \%)$ y dos $(4.2 \%)$ con sensibilidad intermedia a la tetraciclina y al cloranfenicol, respectivamente.

De los 47 aislamientos de SGA sólo se tipificaron 40; en 27 se presentó el serotipo M2 y todas estas cepas presentaron la secuencia de genes que codifica para SpeA. En este grupo se encontraron ocho de las nueve cepas de SGA aisladas de niños con escarlatina. Nueve cepas presentaron el serotipo 10/12; de éstas ninguna fue positiva para SpeA y sólo una cepa M89 fue también SpeA positiva (figura 1).

\section{Discusión}

Al parecer el brote fue dado por una cepa M2. En el Reino Unido se ha asociado la fiebre escarlatina con los tipos M 1,3,4 y $6 .{ }^{16}$ En Estados Unidos de América

\section{Cuadro I \\ Concentraciones mínimas inhibitorias Y SUSCEPTIBILIDAD DE 47 AISLAMIENTOS de Streptococcus pyogenes obtenidos de exudado faríngeo. México, D.F., México, 1999-2000}

\begin{tabular}{|c|c|c|c|c|c|c|}
\hline Antimicrobiano & $\begin{array}{l}\mathrm{CIM}_{50} \\
(\mathrm{mcg} / \mathrm{ml}\end{array}$ & $\begin{array}{c}\mathrm{ClM}_{90} \\
(\mathrm{mcg} / \mathrm{ml})\end{array}$ & $\begin{array}{l}\text { Rango de CIM } \\
\text { (mcg/ml) }\end{array}$ & Susceptible & $\begin{array}{l}\#(\%) \text { Cepas } \\
\text { Intermedio }\end{array}$ & Resistente \\
\hline Penicilina & 0.03 & 0.125 & $0.03-0.125$ & $47(100)$ & $0(0)$ & $0(0)$ \\
\hline Ampicilina & 0.25 & 0.25 & $0.25-0.5$ & $47(100)$ & $0(0)$ & $0(0)$ \\
\hline Eritromicina & 0.25 & 4.0 & $0.25-4.0$ & $30(63.8)$ & ) 11(23.4) & $6(12.8)$ \\
\hline Clindamicina & 0.25 & 0.5 & $0.25-0.5$ & $41(87.2)$ & $6(12.8)$ & $0(0)$ \\
\hline Cefalotin & 2.0 & 2.0 & $2.0-4.0$ & $47(100)$ & $0(0)$ & $0(0)$ \\
\hline Cefuroxima & 2.0 & 4.0 & $2.0-4.0$ & $47(100)$ & $0(0)$ & $0(0)$ \\
\hline Cefotaxima & 4.0 & 4.0 & $4.0-$ & $47(100)$ & $0(0)$ & $0(0)$ \\
\hline Ceftriazona & 4.0 & 4.0 & $4.0-$ & $47(100)$ & $0(0)$ & $0(0)$ \\
\hline Gentamicina & 1.0 & 4.0 & $1.0-16.0$ & $44(93.6)$ & $2(4.2)$ & $1(2.1)$ \\
\hline Tetraciclina & 2.0 & 2.0 & $2.0-4.0$ & $43(91.5)$ & $4(8.5)$ & $0(0)$ \\
\hline A mikacina & 16.0 & 16.0 & $16.0-$ & $47(100)$ & $0(0)$ & $0(0)$ \\
\hline Rifampicina & 1.0 & 1.0 & $1.0-$ & $47(100)$ & $0(0)$ & $0(0)$ \\
\hline Cloranfenicol & \begin{tabular}{|l|l|}
4.0 \\
\end{tabular} & 4.0 & $4.0-8.0$ & $45(95.8)$ & $2(4.2)$ & $0(0)$ \\
\hline
\end{tabular}

* CIM: Concentración inhibitoria mínima

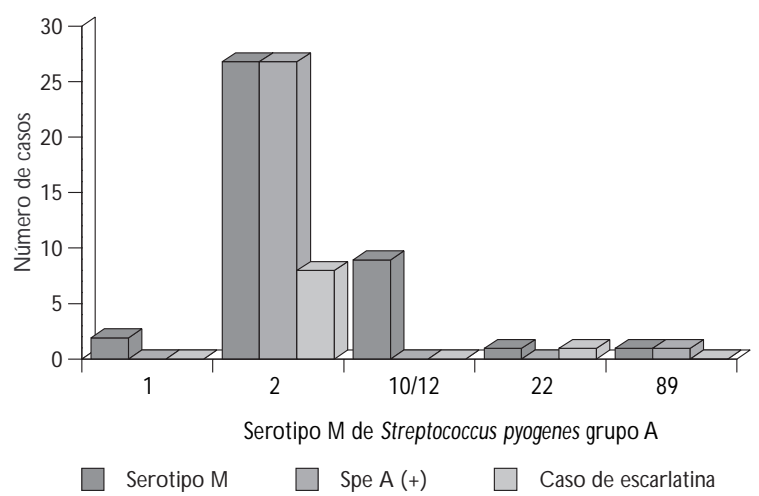

Spe A x Exotoxina pirogénica estreptocóccica A

Figura 1. Distribución de serotipos y presencia de EXotoXINA PIROGÉNICA ESTREPTOCÓ́CCICA A DE $\mathbf{4 0}$ aislamientos de Streptococcus pyogenes, México, D.F., MÉxıCO, 1999-2000

(EUA) el tipo M2 se ha asociado con 9\% de los casos de faringitis no complicada y en menor porcentaje con infección severa y fiebre reumática. ${ }^{17}$ En México, Espinoza de los Monteros y colaboradores ${ }^{18}$ describieron también la caracterización de SGA aislados de un 
brote de escarlatina en una guardería pero con base en el serotipo T. Identificar un serotipo asociado con algún padecimiento producido por SGA no es fácil, principalmente porque los reportes a menudo son de brotes y no de estudios de población. En este caso, a pesar de lo anterior, $100 \%$ de las cepas M2 presentaron SpeA, la principal toxina asociada con la aparición de escarlatina ${ }^{19}$ y sólo una cepa M89 también la presentó; además, ocho de nueve cepas de casos correspondieron a este serotipo. El que no todos los niños con cepa M2 desarrollaran el proceso se debió, como lo explica Stevens ${ }^{20}$ a la condición inmunológica de cada paciente, aunado a la presencia de anticuerpos específicos.

A pesar del tratamiento a los cuatro casos iniciales y a 16 contactos directos, se presentaron ocho casos más de escarlatina y un total de 47 aislamientos de SGA. Se desconoce el porqué de los fallos, ya que no se identificaron cepas resistentes a la penicilina ni a ningún beta lactámico, lo que concuerda con todo lo reportado en la literatura hasta ahora y que reconoce que SGA sigue siendo altamente sensible a la penicilina y sus derivados. ${ }^{21,22}$

Respecto a los otros antibióticos, utilizados frecuentemente como alternativa de tratamiento, aunque el número estudiado es pequeño, los resultados son similares a los reportados con anterioridad por nuestro equipo tanto para beta lactámicos como para eritromicina. ${ }^{23}$ Respecto de esta última se tuvieron seis $(12.7 \%)$ de cepas resistentes; la literatura reporta datos tan variados que van de $1 \%$ de Henning ${ }^{24}$ 9.6\% por nosotros, ${ }^{23} 12.7 \%$ de Arvand, ${ }^{8} 16 \%$ de Rodríguez, ${ }^{22} 27 \%$ de Baquero $^{25}$ y hasta $35.8 \%$ de Melo Cristino ${ }^{26}$ y $78 \%$ de Po-Ren Hsueh. ${ }^{9}$ Las diferencias muy probablemente estén dadas por la frecuencia en el uso del antibiótico, por lo que creemos que sólo debe usarse cuando no esté indicada la penicilina, como en el caso de los pacientes alérgicos, además de realizar antibiograma en estos casos.

Cinco de las seis cepas resistentes a eritromicina correspondieron al serotipo 2. Murayama y colaboradores ${ }^{27}$ refieren que la mayoría de las cepas resistentes a eritromicina pertenecen a un número limitado de serotipos $\mathrm{M}$, además, las seis cepas fueron sensibles a todos los otros antibióticos probados.

No se presentaron cepas resistentes a clindamicina, sin embargo, seis $(12.7 \%)$ tuvieron sensibilidad intermedia. Gene y colaboradores ${ }^{21}$ tampoco encontraron cepas resistentes excepto una, al igual que Kataja. ${ }^{28}$ Respecto al análisis en conjunto de penicilina, clindamicina y eritromicina, los patrones de sensibilidad son similares a los reportados por Espinoza de los Monteros y colaboradores, ${ }^{18}$ y por Rodríguez y colaboradores ${ }^{22}$ quienes informan $100 \%$ de cepas sensibles a penicilina y clindamicina, pero constantemente resistentes a eritromicina, lo que se describe como fenotipo $\mathrm{M}^{3}$ Consideramos importante hacer un seguimiento periódico a la sensibilidad del SGA contra clindamicina, debido a que se ha documentado una mayor efectividad de éste que la misma penicilina en las infecciones invasivas por SGA. ${ }^{5}$

Respecto al análisis de la CIM de los otros antibióticos beta-lactámicos, la totalidad de las cepas fueron sensibles, sin embargo, el valor de la CIM90 es el doble para la ampicilina y bastante más alto para los otros que lo requerido para la penicilina, datos que concuerdan con autores como Connan y colaboradores ${ }^{10}$ y Rodríguez y colaboradores, ${ }^{22}$ aunque sus valores de la CIM90 son menores, lo que comprueba las variantes de la actividad in vitro de estos antibióticos.

La clasificación del Streptococcus pyogenes ha evolucionado desde pruebas de aglutinación y caracterización fenotípica, hasta métodos más sofisticados de tipificación genética. Debido a que la especificidad biológica de la proteína $\mathrm{M}$ es codificada por una región del gen emm de menos de 400 pares de bases, ${ }^{11}$ técnicas como la utilizada en este trabajo permiten caracterizar tipos $\mathrm{M}$ no tipificables serológicamente que pueden representar hasta $20 \%$ del total de cepas estudiadas en una población, como lo refieren Johnson y colaboradores. ${ }^{14}$

\section{Agradecimientos}

Nuestro agradecimiento al Dr. Luis Manuel Perea Mejía, por su asistencia técnica y su invaluable colaboración para la realización de este trabajo, así como a la C. Ofelia Pedreguera, por la elaboración del manuscrito.

\section{Referencias}

1. Kaplan EL.The resurgence of group A streptococcal infections and their sequelae. Eur J Clin Microbiol Infect Dis 1991;10:55-57.

2. Spencer RC. Invasive streptococci. Eur J C lin Microbiol Infect D is 1995; 14(Suppl 1):26-32.

3. Schwartz B, Facklam RR, Breiman RF. C hanging epidemiology of group A streptococcal infection in the U.S.A. Lancet 1990;336:1167-1171.

4. Talkington DF, Schwartz B, Black CM, Todd JK, Elliot J, Breiman RF et al. Association of phenotypic and genotypic characteristics of invasive Streptococcus pyogenes isolates with clinical components of streptococcal toxic shock syndrome. Infect Immun 1993;61:3369-3374.

5. C urtis N . Invasive group A streptococcal infection. C urr O pin Infect D is 1996;9:191-202.

6. Brook I. The role of beta-lactamase producing bacteria in the persistence of streptococcal tonsillar infection. Rev Infect D is 1984;6:601-607. 7. Fitoussi F, Cohen R, Brami G, D oit C, Brahimi N, De la Rocque $F$ et al. Molecular DN A analysis for differentiation of persistence or relapse from 
recurrence in treatment failure of Streptococcus pyogenes pharyngitis. Eur J Clin Microbiol Infect D is 1997;16:233-237.

8.Arvand $\mathrm{M}$, Hoeck M, Hahn $\mathrm{H}$,W agner J.Antimicrobial resistance in Streptococcus pyogenes isolates in Berlin. J Antimicrob Chemother 2000;46(4):621-624. 9. Po-Ren $\mathrm{H}$, Hung-Mo Ch, Ay-Huey H, Jiunn-Jomg W. D ecreased activity of erythromycin against Streptococcus pyogenes in Taiwan. Antimicrob A gents Chemother 1995;39:2239-2242.

10. Connan K, Kaplan EL. In vitro susceptibility of recent N orth American Group A streptococcal isolates to eleven oral antibiotics. Pediatr Infect Dis J 1994;13:630-635.

11. N ational Comitee for C linical Laboratory Standars. Performance standars for antimicrobial disk susceptibility test. Villanova (PA): $\mathrm{N}$ ational $\mathrm{Co-}$ mitte for C linical Laboratory Standars, 1997. N CCLS document M2 A6.

12. N ational Comitte for Clinical Laboratory Standars. Methods for dilution antimicrobial susceptibility test for bacteria that grow aerobically. Villanova (PA): N ational Comitee For C linical Laboratory Standars, 1997. NCCLS document M7 A4.

13. Perea-Mejía LM, Stockbauer KE, Pan X, Cravioto A, Musser JM. Characterization of group $A$ Streptococcus strains recovered from Mexican children with pharyngitis by automated DNA sequencing of virulencerelated genes: Unexpectedly large variation in the gene (sic) encoding a complement-inhibiting protein. J Clin Microbiol 1997;35:3220-3224.

14.W hatmore AM, Kapur V, Sullivan J, Musser JM, Kehoe MA. N on-congruent relationships between variation in emm gene sequences and the population genetic structure of group A streptococci. Mol Microbiol 1994; 14:619-631. 15. Beall B, Facklam R, Thompson T. Sequencing emm-specific PCR products for routine and accurate typing of group A streptococci.] C lin Microbiol 1996;34(4):953-958.

16. G aworzewska E, Colman G. Changes in the pattern of infection caused by Streptococcus pyogenes. Epidemiol Infect 1988;100:257-269.

17. Johnson DR, Stevens DL, Kaplan EL. Epidemio logic analysis of group A streptococcal serotypes associated with severe systemic infections, rheumatic fever, or uncomplicated pharyngitis. J Infect $D$ is 1992;166:374-382. 18. Espinoza-de los Monteros LE, Bustos IM, Flores LV, Avila-Figueroa C. 0 utbreak of scarlet fever caused by an erythromycin-resistant Strepto- coccus pyogenes emm 22 genotype strain in a day-care-center. Pediatr Infect Dis J 2001;20(8):807-809.

19. Shiseki M, Miwa K, N emoto Y, Kato H, Susuki J, Sekiya K et al.Comparison of pathogenic factors expressed by group A streptococci isolated from patients with streptococcal toxic shock syndrome and scarlet fever. Microb Pathog 1999;27(4):243-252.

20. Stevens D L. Invasive group A streptococcal infections:The past, present and future. Pediatr Infect Dis J 1994;13:561-566.

21. Gené A, G onzález-C uevas A, Juncosa T, Luaces C , Latorre C. Sensibilidad antibiótica de Streptococcus pyogenes en pediatría. Enferm Infecc Microbiol Clin 1998;16:272-274.

22. Rodríguez RS, Calderón-Jaimes E, Gómez-Barreto D, Espinosa-de los Monteros LE. Antimicrobial resistance characteristics of clinical isolates of Streptococcus pyogenes. Salud Publica Mex 2000;42(3):226-229.

23. González-Pedraza AA, 0 rtiz-Zaragoza MC. Streptococcus pyogenes: susceptibilidad in vitro y papel de las bacterias productoras de betalactamasa en la persistencia de la faringoamigdalitis estreptocóccica.Aten Primaria 2000;25:542-545.

24. Henning $C$, Bengtsson L, Jorup $C$, Engquist $S$. Antibiotic resistance in Streptococcus pneumoniae, Haemophilus influenzae and Streptococcus pyogenes in respiratory tract infections in outpatients. Scand J Infect D is 1997; 29(6):559-563.

25. Baquero F, García-Rodríguez JA, García-de Lomas, A guilar L. Antimicrobial resistance of 914 beta-hemolytic streptococci isolated from pharyngeal swabs in Spain: Results of a 1-year (1996-1997) multicenter surveillance study. Antimicrob A gents Chemother 1999;43(1):178-180.

26. Melo-Cristino J, Fernández ML. Streptococcus pyogenes isolated in Portugal: Macrolide resistance phenotypes and correlation with T types. Portuguese surveillance group for the study of respiratory pathogens. Microb Drug Resist 1999;5(3):219-225.

27. Murayama S, Yoshioka H, Fojita K, Takimoto M, Satake Y. Sensitivity of group A streptococci to antibiotics. Am J D is Child 1979;133:1143-1145. 28. Kataja J, Houvinen P, Skurnik M, Seppälä H. Erythromycin resistance in group A streptococci in Finland.The Finnish study group for antimicrobial resistance.Antimicrob A gents Chemother 1999;43(1):48-52. 